PROCEEDINGS OF THE AMERICAN MATHEMATICAL SOCIETY

Volume 124, Number 1, January 1996

\title{
SINGLE-POINT CONDENSATION AND LEAST-ENERGY SOLUTIONS
}

\author{
XIAOFENG REN AND JUNCHENG WEI \\ (Communicated by Jeffrey Rauch)
}

\begin{abstract}
We prove a conjecture raised in our earlier paper which says that the least-energy solutions to a two-dimensional semilinear problem exhibit single-point condensation phenomena as the nonlinear exponent gets large. Our method is based on a sharp form of a well-known borderline case of the Sobolev embedding theory. With the help of this embedding, we can use the Moser iteration scheme to carefully estimate the upper bound of the solutions. We can also determine the location of the condensation points.
\end{abstract}

\section{INTRODUCTION}

In this work, we shall confirm the conjecture raised in our earlier paper [8]. We study

$$
\left\{\begin{array}{l}
\Delta u+u^{p}=0 \text { in } \Omega, \\
u=0 \text { on } \partial \Omega
\end{array}\right.
$$

where $p>1$ and $\Omega$ is a smooth bounded domain in $R^{2}$. We consider the so-called least-energy solutions of (1.1) obtained by minimizing the functional

$$
J_{p}:\left\{v \in W_{0}^{1,2}(\Omega):\|v\|_{p+1}=1\right\} \rightarrow R
$$

where $J_{p}(v)=\int_{\Omega}|\nabla v|^{2}$. Standard variational arguments show that the minimum of $J_{p}$ is achieved by a positive function in $\left\{v \in W_{0}^{1,2}(\Omega):\|v\|_{p+1}=1\right\}$, and a positive scalar multiple of the function solves (1.1). Let us denote such a leastenergy solution by $u_{p}$. In [8] we proved

Theorem 1.1. There exist $C_{1}, C_{2}$, independent of $p$, such that

$$
0<C_{1}<\left\|u_{p}\right\|_{L^{\infty}(\Omega)}<C_{2}<\infty
$$

for $p$ large enough.

To understand the shape of $u_{p}$ for large $p$, let

$$
v_{p}=\frac{u_{p}}{\int_{\Omega} u_{p}^{p}}
$$

Received by the editors July 2, 1994.

1991 Mathematics Subject Classification. Primary 35B40, 35A08, 35A15; Secondary 34A34.

(C)1996 American Mathematical Society 
For a sequence $v_{p_{n}}$ of $v_{p}$ we define the blow-up set $S$ of $v_{p_{n}}$ to be the subset of $\bar{\Omega}$ such that $x \in S$ if there exist a subsequence, still denoted by $v_{p_{n}}$, and a sequence $x_{n}$ in $\Omega$ with

$$
v_{p_{n}}\left(x_{n}\right) \rightarrow \infty \text { and } x_{n} \rightarrow x
$$

We use $\# S$ to denote the cardinality of $S$. We also define a peak point $P$ for $u_{p}$ to be a point in $\bar{\Omega}$ such that $u_{p}$ doesn't vanish in the $L^{\infty}$ norm in any small neighborhood of $P$ as $p \rightarrow \infty$. It turns out later that the set of the peak points of $u_{p}$ is the same as the set of blow-up points of $v_{p}$. About the blow-up set of $v_{p}$ we proved the following theorem in [8].

Theorem 1.2. 0 For any smooth bounded domain $\Omega$ and a sequence $v_{p_{n}}$ of $v_{p}$ with $p_{n} \rightarrow \infty$ there exists a subsequence of $v_{p_{n}}$ such that the blow-up set $S$ of that subsequence is contained in $\Omega$ and has the property $1 \leq \# S \leq 2$; in other words the subsequence must blow up, and it blows up at most at two points in $\Omega$.

(2) If $\Omega$ meets condition $(\mathrm{T})$, then the above subsequence must blow up at one point in $\Omega$.

Here condition $(\mathrm{T})$ is a geometric condition on $\Omega$. A domain $\Omega$ is said to satisfy condition (T) if (A) $\Omega$ is star-shaped with respect to some point $y \in \Omega$, i.e., $(x-$ $y, n(x))>0$ for all $x \in \partial \Omega$, and (B)

$$
\int_{\partial \Omega} \frac{d s}{(x-y, n(x))}<2 \pi e
$$

where $n(x)$ denotes the outer normal of $\partial \Omega$ at $x$.

About the location of the blow-up points, we proved in [8]

Theorem 1.3. Let $\Omega$ be a smooth bounded domain with condition (T). Then for any sequence $v_{p_{n}}$ of $v_{p}$ with $p_{n} \rightarrow \infty$ there exists a subsequence of $v_{p_{n}}$, still denoted by $v_{p_{n}}$, such that

(1)

$$
f_{n}:=\frac{u_{p_{n}}^{p_{n}}}{\int_{\Omega} u_{p_{n}}^{p_{n}}}=\left(\int_{\Omega} u_{p_{n}}^{p_{n}}\right)^{p_{n}-1} v_{p_{n}}^{p_{n}} \rightarrow \delta\left(x_{0}\right)
$$

in the sense of distribution where $\delta\left(x_{0}\right)$ is the $\delta$ function at point $x_{0}$ and $\left\{x_{0}\right\}=S$.

(2) $v_{p_{n}} \rightarrow G\left(x, x_{0}\right)$ in $W^{1, q}(\Omega)$ weakly for any $1<q<2$ where $G$ is the Green's function of $-\Delta$ on $\Omega$; furthermore for any compact subset $K$ of $\bar{\Omega} \backslash\left\{x_{0}\right\}$ we have $v_{p_{n}} \rightarrow G\left(x, x_{0}\right)$ in $C^{2, \alpha}(K)$.

(3) $x_{0}$ is a critical point of function $\phi$ where $\phi(x)=g(x, x)$ and

$$
g(x, y)=G(x, y)+\frac{1}{2 \pi} \log |x-y|
$$

is the regular part of the Green's function $G$.

At the end of [8], we conjectured that condition (T) is unnecessary for Theorem 1.2 (2) and Theorem 1.3, i.e., least-energy solutions must develop single peaks regardless of domains. In this paper we shall prove

Theorem 1.4. $1 \leq \liminf _{p \rightarrow \infty}\left\|u_{p}\right\|_{L^{\infty}(\Omega)} \leq \limsup _{p \rightarrow \infty}\left\|u_{p}\right\|_{L^{\infty}(\Omega)} \leq \sqrt{e}$. 
Theorem 1.5. For any smooth bounded domain $\Omega$ and a sequence $v_{p_{n}}$ of $v_{p}$ with $p_{n} \rightarrow \infty$ there exists a subsequence of $v_{p_{n}}$ such that the blow-up set $S$ of that subsequence contains only one point in $\Omega$; in other words, the subsequence must blow up and it blows up at one point.

Theorem 1.6. The conclusions in Theorem 1.3 are true without condition (T).

From the above results, we see that when $p$ gets large, the least-energy solutions $u_{p}$ look more and more like a single spike.

If we consider a convex domain $\Omega$, then, using a result of L. Caffarelli and A. Friedman (Theorem 3.1 of [3]) which implies that $\phi$ is strictly convex, $\phi$ has strictly positive Hessian and the only critical point of $\phi$ is the global minimum, we have

Corollary 1.7. For convex domain $\Omega$ the conclusions of Theorem 1.5 and Theorem 1.6 are true for the whole families $\left\{u_{p}\right\},\left\{v_{p}\right\}$ and $\left\{f_{p}\right\}$ as $p \rightarrow \infty$, and $x_{0}$ is the unique critical point of $\phi$.

Remark 1.8. It was shown by C.-S. Lin [7] that for convex domain $\Omega$ the solution $u_{p}$ of (1.1) minimizing (1.2) is unique.

Some techniques in this paper have been extended to the $N$-dimensional case where the Laplacian operator is replaced by the $N$-Laplacian operator. See [9] for details. The mixed boundary version of the two-dimensional problem is also considered by the authors in [10]. In this paper we shall prove Theorem 1.4 in section 2 and Theorem 1.5 in section 3. We omit the proof of Theorem 1.6 since it is identical to the proof of Theorem 1.3 [8].

\section{Proof of Theorem 1.4}

Define

$$
c_{p}:=\inf \left\{\left[\int_{\Omega}|\nabla u|^{2}\right]^{1 / 2}: u \in W_{0}^{1,2}(\Omega),\|u\|_{p+1}=1\right\} .
$$

We collect some results from [8] that will lead to the asymptotic behavior of $c_{p}$.

Lemma 2.1. For every $t \geq 2$ there is $D_{t}$ such that

$$
\|u\|_{L^{t}} \leq D_{t} t^{1 / 2}\|\nabla u\|_{L^{2}}
$$

for all $u \in W_{0}^{1,2}(\Omega)$ where $\Omega$ is a bounded domain in $R^{2}$; furthermore

Lemma 2.2 .

$$
\lim _{t \rightarrow \infty} D_{t}=(8 \pi e)^{-1 / 2} \text {. }
$$

\section{Corollary 2.3.}

$$
\lim _{p \rightarrow \infty} \frac{c_{p}}{p^{-1 / 2}}=(8 \pi e)^{1 / 2}
$$

$$
\lim _{p \rightarrow \infty} p \int_{\Omega} u_{p}^{p+1}=8 \pi e, \lim _{p \rightarrow \infty} p \int_{\Omega}\left|\nabla u_{p}\right|^{2}=8 \pi e .
$$

We now define an important quantity

$$
L_{0}=\varlimsup_{p \rightarrow \infty} \frac{p \nu_{p}}{2 \sqrt{e}}
$$

where

$$
\nu_{p}=\int_{\Omega} u_{p}^{p}
$$


Notice that the quantity $L_{0}$ defined here is different from the one in [8]. Indeed this difference will lead to Theorem 1.5.

We have the following estimate for $L_{0}$.

Corollary 2.4. For any smooth bounded domain $\Omega$ of $R^{2}, L_{0} \leq 4 \pi \sqrt{e}$.

The proofs of these facts can be found in section 2 of [8].

Now let us start the proof of Theorem 1.4. A uniform lower bound actually exists for any positive solutions to (1.1). Let $\lambda$ be the first eigenvalue of $-\Delta$ and $\varphi$ be a corresponding positive eigenfunction. Then if $u$ is any solution to (1.2) with the exponent equal to $p$, we have

$$
0=\int_{\Omega} u \Delta \varphi-\varphi \Delta u=-\lambda \int_{\Omega} u \varphi+\int_{\Omega} u^{p} \varphi .
$$

That is, $\int_{\Omega}\left(u^{p}-\lambda u\right) \varphi=0$. Hence $\|u\|_{L^{\infty}(\Omega)} \geq \lambda^{1 /(p-1)} \rightarrow 1$ as $p \rightarrow \infty$ which yields a lower bound for large $p$.

To get an upper bound for $\left\{u_{p}\right\}$, we use an iteration argument. Fix positive $\alpha$ and $\epsilon$ that will be chosen small later. Letting $\nu=(1+\alpha)(p+1)$, from Lemma 2.1, we have

$$
\left[\int_{\Omega} u_{p}^{\nu}\right]^{1 / \nu} \leq(8 \pi e)^{-1 / 2} E_{(1+\alpha)(p+1)} \nu^{1 / 2}\left\|\nabla u_{p}\right\|_{L^{2}(\Omega)}
$$

where $\lim _{p \rightarrow \infty} E_{(1+\alpha)(p+1)}=1$. But from Corollary 2.3, we know

$$
\lim _{p \rightarrow \infty} p \int_{\Omega}\left|\nabla u_{p}\right|^{2}=8 \pi e .
$$

Hence there is $P_{0}$ such that for all $p>P_{0}$,

$$
\left(\int_{\Omega} u_{p}^{\nu}\right)^{2 / \nu} \leq 1+\alpha+\epsilon, \text { or } \int_{\Omega} u_{p}^{\nu} \leq(1+\alpha+\epsilon)^{\nu / 2} .
$$

We henceforth only consider $p>P_{0}$.

Multiplying both sides of (1.1) by $u_{p}^{2 s-1}$, we get, after integrating by parts,

$$
\frac{2 s-1}{s^{2}} \int_{\Omega}\left|\nabla u_{p}^{s}\right|^{2}=\int_{\Omega} u_{p}^{p-1+2 s} .
$$

Using Lemma 2.1 again, we deduce

$$
\begin{gathered}
{\left[\int_{\Omega} u_{p}^{\nu s}\right]^{1 / \nu} \leq D_{\nu s} \nu^{1 / 2}\left\|\nabla u_{p}^{s}\right\|_{L^{2}(\Omega)}} \\
{\left[\int_{\Omega} u_{p}^{\nu s}\right]^{2 / \nu} \leq C_{0} \nu \frac{s^{2}}{2 s-1} \int_{\Omega} u_{p}^{p-1+2 s} \leq C_{1} \nu s \int_{\Omega} u_{p}^{p-1+2 s}}
\end{gathered}
$$

where $D_{\nu s}$ is defined in Lemma 2.1 and $C_{0}$ and $C_{1}$ are constants independent of $p>P_{0}$. Hence we have

$$
\left[\int_{\Omega} u_{p}^{\nu s}\right]^{2 / \nu} \leq C_{1} \nu s \int_{\Omega} u_{p}^{p-1+2 s} .
$$

We now define two sequences $\left\{s_{j}\right\}$ and $\left\{M_{j}\right\}$ by

$$
\begin{array}{ll}
p-1+2 s_{0}=\nu, & p-1+2 s_{j+1}=\nu s_{j} ; \\
M_{0}=(1+\alpha+\epsilon)^{\nu / 2}, & M_{j+1}=\left[C_{1} \nu s_{j} M_{j}\right]^{\nu / 2}
\end{array}
$$

where $C_{1}$ is the constant in (2.6). From (2.4) and (2.6), we have

$$
\int_{\Omega} u_{p}^{\nu s_{j-1}} \leq M_{j}
$$


Next we claim

$$
M_{j} \leq \exp \left[m(\alpha, p, \epsilon) \nu s_{j-1}\right]
$$

where $m(\alpha, p, \epsilon)$ is a constant depending on $\alpha, p$ and

$$
\lim _{p \rightarrow \infty} m(\alpha, p, \epsilon)=\frac{1+\alpha}{2 \alpha} \log (1+\alpha+\epsilon) .
$$

In fact, we can write down $\left\{s_{j}\right\}$ explicitly as

$$
s_{j}=\frac{1}{\nu-2}\left\{\left(\frac{\nu}{2}\right)^{j+1}(\nu-p-1)+p-1\right\} .
$$

Put

Hence

$$
\sigma_{j}=\frac{\nu}{2} \log \left(C_{1} \nu s_{j}\right), \mu_{j}=\log M_{j}
$$

Therefore

$$
\mu_{j+1}=\frac{\mu_{j}}{2}+\sigma_{j}
$$

$$
\begin{gathered}
\sigma_{j}=\frac{\nu}{2}\left\{\log \left[\frac{C_{1} \nu}{\nu-2}\right]+\log \left[\left(\frac{\nu}{2}\right)^{j+1}(\nu-p-1)+p-1\right]\right\} \\
\leq\left[\nu \log \sqrt{2 C_{1}} \nu\right](j+1) .
\end{gathered}
$$

Now we define $\left\{\tau_{j}\right\}$ by

$$
\tau_{0}=\mu_{0}, \tau_{j+1}=\frac{1}{2} \nu \tau_{j}+\left(\nu \log \sqrt{2 C_{1}} \nu\right)(j+1) .
$$

Clearly $\mu_{j} \leq \tau_{j}$. Moreover we have

$$
\begin{gathered}
\tau_{j}=\left(\frac{\nu}{2}\right)^{j}\left[\mu_{0}+2 \nu \log \left(\sqrt{2 C_{1}} \nu\right) \frac{\nu}{(\nu-2)^{2}}\right]-\frac{2}{\nu-2}\left[\nu \log \left(\sqrt{2 C_{1}} \nu\right)\left(j+\frac{\nu}{\nu-2}\right)\right] \\
\leq \frac{\mu_{0}+2 \nu \log \left(\sqrt{2 C_{1}} \nu\right) \frac{\nu}{(\nu-2)^{2}}}{(\nu-2)^{-1}(\nu-p-1)} s_{j-1} \\
\leq \frac{\mu_{0}+2 \nu \log \left(\sqrt{2 C_{1}} \nu\right) \frac{\nu}{(\nu-1)^{2}}}{\nu-p-1} \frac{\nu-2}{\nu} \nu s_{j-1}:=m(\alpha, p, \epsilon) \nu s_{j-1}
\end{gathered}
$$

where

$$
\lim _{p \rightarrow \infty} m(\alpha, p, \epsilon)=\frac{1+\alpha}{2 \alpha} \log (1+\alpha+\epsilon)
$$

Remember $\nu=(1+\alpha)(p+1)$. This proves $(2.9)$. Therefore we get

$$
\left\|u_{p}\right\|_{L^{\nu s_{j-1}(\Omega)}} \leq \exp [m(\alpha, p, \epsilon)] .
$$

Sending $j \rightarrow \infty$ and then $p \rightarrow \infty$, we deduce

$$
\limsup _{p \rightarrow \infty}\left\|u_{p}\right\|_{L^{\infty}} \leq(1+\alpha+\epsilon)^{\frac{1+\alpha}{2 \alpha}} .
$$

Sending $\epsilon, \alpha \rightarrow 0$, we deduce

$$
\limsup _{p \rightarrow \infty}\left\|u_{p}\right\|_{L^{\infty}} \leq \sqrt{e}
$$

We include a consequence of Theorem 1.4 here which will be used later.

Corollary 2.5. There exist $C_{1}$ and $C_{2}$ such that

$$
\frac{C_{1}}{p} \leq \int_{\Omega} u_{p}^{p} \leq \frac{C_{2}}{p}
$$


Proof. The first inequality follows from Theorem 1.4 and the first limit of Corollary 2.3; the second inequality follows from the first limit of Corollary 2.3 through an interpolation argument.

\section{Proof of Theorem 1.5}

The proof is similar to the proof of Theorem 1.2 in [8]. The major difference appears when we reach (3.7). We first state a boundary estimate lemma. The proof of the lemma is standard. One combines the moving plane method in [5] with a Kelvin transform. We refer to [4] and [5] for details.

Lemma 3.1. Let $u$ be a solution of

$$
\left\{\begin{array}{l}
\Delta u+f(u)=0 \text { in } \Omega \subset R^{2}, \\
\left.u\right|_{\partial \Omega}=0, u>0 \text { in } \Omega
\end{array}\right.
$$

where $\Omega$ is bounded, smooth and $f$ is a smooth function. Then there exist a neighborhood $\omega$ of $\partial \Omega$ and a constant $C$ both depending on the geometry of $\Omega$ only such that

$$
\|u\|_{L^{\infty}(\omega)} \leq C\|u\|_{L^{1}(\Omega)} .
$$

Applying this lemma to

$$
v_{p}=\frac{u_{p}}{\int_{\Omega} u_{p}^{p}}
$$

we have the following uniform boundary estimate. In particular, it implies that $\left\{v_{p}\right\}$ doesn't blow up on the boundary of $\Omega$; hence by Corollary $2.5\left\{u_{p}\right\}$ has no peak on the boundary of $\Omega$.

Lemma 3.2. There exist a constant $C$ and a neighborhood $\omega$ of $\partial \Omega$ both depending on the geometry of $\Omega$ only such that $v_{p} \leq C$ in $\omega$.

Proof. Because $\left\|\Delta v_{p}\right\|_{L^{1}}=1$, combining the elliptic $L^{p}$ estimate with the duality argument (see [2] for details), we have that $v_{p}$ is bounded uniformly in $W^{1, q}(\Omega)$ for $1 \leq q<2$; hence $v_{p}$ is uniformly bounded in $L^{1}$. Then using Lemma 3.1, we obtain the desired result.

Lemma 3.3. Let $S$ be the blow-up set defined in (1.4) of a subsequence $v_{n}$ of $v_{p}$. Then $S$ is nonempty and there is a small neighborhood $\omega$ of $\partial \Omega$ which depends on the geometry of $\Omega$ only such that $S \cap \omega=\emptyset$.

Proof. The second assertion follows immediately from Lemma 3.2. For the first one, observe

$$
\max v_{n}(x) \geq \frac{C}{\nu_{p_{n}}} \rightarrow \infty
$$

from Theorem 1.4, Corollary 2.5 and the definition of $v_{n},(1.3)$.

We quote an interesting $L^{1}$ estimate from [1].

Lemma 3.4. Let $u$ be a solution of

$$
\left\{\begin{array}{l}
-\Delta u=f \text { in } \Omega \\
\left.u\right|_{\partial \Omega}=0
\end{array}\right.
$$

where $\Omega$ is a smooth bounded domain in $R^{2}$. We have for $0<\epsilon<4 \pi$

$$
\int_{\Omega} \exp \left[\frac{(4 \pi-\epsilon)|u(x)|}{\|f\|_{L^{1}}}\right] d x \leq \frac{4 \pi^{2}}{\epsilon}(\operatorname{diam} \Omega)^{2} .
$$


Now recall

$$
L_{0}=\varlimsup_{p \rightarrow \infty} \frac{p \nu_{p}}{2 \sqrt{e}} \leq 4 \pi \sqrt{e}
$$

where $\nu_{p}=\int_{\Omega} u_{p}^{p}$.

We denote any sequence $u_{p_{n}}$ of $u_{p}$ by $u_{n}$. Because $u_{n}$ has property

$$
\int_{\Omega} \frac{u_{n}^{p_{n}}}{\int_{\Omega} u_{n}^{p_{n}}}=1
$$

we can extract a subsequence of $u_{n}$, still denoted by $u_{n}$, so that there is a positive bounded measure $\mu$ in $M(\Omega)$, the set of all real bounded Borel measures on $\Omega$, such that

$$
\int_{\Omega} f_{n} \varphi \rightarrow \int_{\Omega} \varphi d \mu
$$

for all $\varphi \in C_{0}(\Omega)$ where

$$
f_{n}=\nu_{p_{n}}^{p_{n}-1} v_{n}^{p_{n}} \text { and } v_{n}=\frac{u_{n}}{\nu_{p_{n}}}
$$

For any $\delta>0$, we call $x_{0}$ a $\delta$-regular point if there is a function $\varphi$ in $C_{0}(\Omega)$, $0 \leq \varphi \leq 1$, with $\varphi=1$ in a neighborhood of $x_{0}$ such that

$$
\int_{\Omega} \varphi d \mu<\frac{4 \pi}{L_{0}+2 \delta}
$$

We define $\Sigma(\delta)=\left\{x_{0} \in \Omega: x_{0}\right.$ is not a $\delta$-regular point $\}$. Clearly

$$
\mu\left(x_{0}\right) \geq \frac{4 \pi}{L_{0}+2 \delta}
$$

for all $x_{0} \in \Sigma(\delta)$.

Our next lemma plays a central role in the proof of Theorem 1.5. It says that smallness of $\mu$ at a point $x_{0}$ implies boundedness of $v_{n}$ near $x_{0}$.

Lemma 3.5. If $x_{0}$ is a $\delta$-regular point, then $\left\{v_{n}\right\}$ is bounded in $L^{\infty}\left(B_{R_{0}}\left(x_{0}\right)\right)$ for some $R_{0}>0$.

Proof. Let $x_{0}$ be a $\delta$-regular point. From the definition of $\delta$-regular points, there exists $R_{1}>0$ such that

Split $v_{n}$ into two parts

$$
\int_{B_{R_{1}}\left(x_{0}\right)} f_{n}<\frac{4 \pi}{L_{0}+\delta}
$$

where $v_{1 n}$ is the solution of

$$
v_{n}=v_{1 n}+v_{2 n}
$$

$$
\left\{\begin{array}{l}
\Delta v_{1 n}+f_{n}=0 \text { in } B_{R_{1}}\left(x_{0}\right) \\
\left.v_{1 n}\right|_{\partial B_{R_{1}}\left(x_{0}\right)}=0
\end{array}\right.
$$

and $v_{2 n}$ solves

$$
\left\{\begin{array}{l}
\Delta v_{2 n}=0 \text { in } B_{R_{1}}\left(x_{0}\right) \\
\left.v_{2 n}\right|_{\partial B_{R_{1}}\left(x_{0}\right)}=\left.v_{n}\right|_{\partial B_{R_{1}}\left(x_{0}\right)} .
\end{array}\right.
$$

From the maximum principle, $v_{1 n}, v_{2 n}>0$. By the mean value theorem for harmonic functions, we have

$$
\left\|v_{2 n}\right\|_{L^{\infty}\left(B_{R_{1} / 2}\right)} \leq C\left\|v_{2 n}\right\|_{L^{1}\left(B_{R_{1}}\right)} \leq C\left\|v_{n}\right\|_{L^{1}(\Omega)} \leq C
$$


where the last inequality follows as in the proof of Lemma 3.2. So we need only to consider $v_{1 n}$.

We first claim that when $n$ is large enough

$$
f_{n}(x) \leq \exp \left(L_{0}+\delta / 2\right) v_{n}(x)
$$

for all $x \in \Omega$. Observe that

$$
\limsup _{p \rightarrow \infty}\left\|u_{p}\right\|_{L^{\infty}(\omega)} \leq \sqrt{e}, \lim _{p \rightarrow \infty}\left(\int_{\Omega} u_{p}\right)^{1 / p}=1
$$

from Theorem 1.4 and Corollary 2.5. Therefore

$$
\limsup _{p \rightarrow \infty} \frac{\left\|u_{p}\right\|_{L^{\infty}(\omega)}}{\left(\int_{\Omega} u_{p}\right)^{1 / p}} \leq \sqrt{e}<e .
$$

Let

$$
\alpha_{n}=\frac{\left\|u_{p_{n}}\right\|_{L^{\infty}(\omega)}}{\left(\int_{\Omega} u_{p_{n}}\right)^{1 / p_{n}}} .
$$

Then

$$
\limsup _{n \rightarrow \infty} \alpha_{n}<e .
$$

Consider the function $\log x / x$ which is increasing if $x<e$. Since for every $x \in \Omega$

$$
\frac{u_{n}(x)}{\nu_{n}^{1 / p_{n}}} \leq \alpha_{n}
$$

fixing small $\epsilon$, we have for large $n$,

$$
\frac{\log \frac{u_{p_{n}}(x)}{\nu_{n}^{1 / p_{n}}}}{\frac{u_{p_{n}}(x)}{\nu_{n}^{1 / p_{n}}}} \leq \frac{\log \alpha_{n}}{\alpha_{n}} \leq \frac{1}{2 \sqrt{e}}+\epsilon .
$$

Therefore

$$
\begin{aligned}
& f_{n}(x) \leq \exp \left[\frac{p_{n} u_{p_{n}}}{\nu_{p_{n}}^{1 / p_{n}}}\left(\frac{1}{2 \sqrt{e}}+\epsilon\right)\right]=\exp \left[\left(\frac{1}{2 \sqrt{e}}+\epsilon\right) p_{n} \nu_{p_{n}}^{1-\frac{1}{p_{n}}} v_{n}\right] \\
\leq & \exp \left[\left(\frac{1}{2 \sqrt{e}}+2 \epsilon\right)\left(\limsup _{n \rightarrow \infty} p_{n} \nu_{p_{n}}\right) v_{n}\right]=\exp \left[\left(L_{0}+4 \sqrt{e} L_{0} \epsilon\right) v_{n}\right]
\end{aligned}
$$

for large $n$. If we choose $\epsilon$ small enough, we have

$$
f_{n}(x) \leq \exp \left[\left(L_{0}+\frac{\delta}{2}\right) v_{n}(x)\right]
$$

for large $n$.

Next we claim that $\left\{f_{n}\right\}$ is uniformly bounded in $L^{1+\delta_{0}}\left(B_{R_{1}} / 2\right)$ for $\delta_{0}$ sufficiently small. Because $\left\{v_{2 n}\right\}$ is uniformly bounded in $B_{R_{1} / 2}\left(x_{0}\right)$, we see from the previous claim

$$
\begin{aligned}
\int_{B_{R_{1}} / 2} f_{n}^{1+\delta_{0}} & \leq \int_{B_{R_{1} / 2}} \exp \left[\left(1+\delta_{0}\right)\left(L_{0}+0.5 \delta\right) v_{n}\right] \\
& \leq C \int_{B_{R_{1}} / 2} \exp \left[\left(1+\delta_{0}\right)\left(L_{0}+0.5 \delta\right) v_{1 n}\right] \\
& \leq C \int_{B_{R_{1} / 2}} \exp \frac{4 \pi\left(1+\delta_{0}\right) \frac{L_{0}+0.5 \delta}{L_{0}+\delta} v_{1 n}}{\int_{B_{R_{1} / 2}\left(x_{0}\right)} f_{n}} \leq C^{\prime}
\end{aligned}
$$


with the aid of Lemma 3.3 if we choose $\delta_{0}$ sufficiently small. So we have proved the claim.

Now take $B_{R_{1} / 4}\left(x_{0}\right)$. We conclude from the weak Harnack inequality (Theorem $8.17,[6])$,

$$
\left\|v_{n}\right\|_{L^{\infty}\left(B_{R_{1} / 4}\left(x_{0}\right)\right)} \leq C\left[\left\|v_{n}\right\|_{L^{2}\left(B_{R_{1} / 2}\left(x_{0}\right)\right)}+\left\|f_{n}\right\|_{L^{1+\delta_{0}}\left(B_{\left.R_{1} / 2\left(x_{0}\right)\right)}\right.}\right] \leq C .
$$

Here the boundedness of $\left\{v_{n}\right\}$ in $L^{2}\left(B_{R_{1} / 2}\left(x_{0}\right)\right)$ follows again from Lemma 3.3.

We now start to prove Theorem 1.5. We first claim $S=\Sigma(\delta)$ for any $\delta>0$. Clearly $S \subset \Sigma$. In fact, let $x_{0} \notin \Sigma$; then $x_{0}$ is a $\delta$-regular point. Hence by Lemma 3.5, $\left\{v_{n}\right\}$ is bounded in $L^{\infty}\left(B_{R}\left(x_{0}\right)\right)$ for some $R$, i.e., $x_{0} \notin S$. Conversely suppose $x_{0} \in \Sigma$. Then we have for every $R>0$, passing to a subsequence of $\left\{v_{n}\right\}$ if necessary,

$$
\lim _{n \rightarrow \infty}\left\|v_{n}\right\|_{L^{\infty}\left(B_{R}\left(x_{0}\right)\right)}=\infty .
$$

Otherwise there would be some $R_{0}>0$ such that $\left\|v_{n}\right\|_{L^{\infty}\left(B_{R_{0}}\left(x_{0}\right)\right)}<C$ for some $C$ independent of $n$. Then

$$
f_{n}=\nu_{n}^{p_{n}-1} v_{n}^{p_{n}} \leq\left(\frac{M}{p_{n}}\right)^{p_{n}-1} C^{p_{n}} \rightarrow 0
$$

uniformly as $n \rightarrow \infty$ on $B_{R_{0}\left(x_{0}\right)}$. Then

$$
\int_{B_{R_{0}}\left(x_{0}\right)} f_{n}=\int_{B_{R_{0}}\left(x_{0}\right)} \nu_{n}^{p_{n}-1} v_{n}^{p_{n}} \leq \epsilon_{0}<\frac{4 \pi}{L_{0}+2 \delta},
$$

which implies that $x_{0}$ is a $\delta$-regular point, i.e., $x_{0} \notin \Sigma$. This contradiction implies (3.8); hence by definition (1.4) of $S, x_{0} \in S$. This completes the proof of our claim.

Next we go back to measure $\mu$ defined in (3.1). Clearly by (3.3)

$$
1 \geq \mu(\Omega) \geq \frac{4 \pi}{L_{0}+2 \delta} \# \Sigma(\delta)=\frac{4 \pi}{L_{0}+2 \delta} \# S .
$$

Hence combining this with Lemma 3.4

$$
1 \leq \# S \leq \frac{L_{0}+2 \delta}{4 \pi} .
$$

Applying Corollary 2.4, we finally conclude that, by choosing $\delta$ small, $\# S=1$.

Remark 3.6. The fact that the peak set of $\left\{u_{n}\right\}$ is included in the blow-up set $S$ of $\left\{v_{n}\right\}$ follows easily from the fact that $\nu_{n} \rightarrow 0$ as $n \rightarrow \infty$. Because the blow-up set contains only one point and the peak set is nonempty from Theorem 1.1, we conclude that the peak set is identical with the blow-up set.

\section{REFERENCES}

1. H. Brezis and F. Merle, Uniform estimate and blow-up behavior for solutions of $-\Delta u=$ $V(x) e^{u}$ in two dimensions, Comm. Partial Differential Equations 16 (1991), 1223-1253. MR 92m:35084

2. H. Brezis and W. Strauss, Semilinear second-order elliptic equations in $L^{1}$, J. Math. Soc. Japan 25 (1973), 565-590. MR 49:826

3. L. Caffarelli and A. Friedman, Convexity of solutions of semilinear elliptic equations, Duke Math. J. 52 (1985), 431-456. MR 87a:35028

4. D. G. DeFigueiredo, P. L. Lions and R. D. Nussbaum, A priori estimates and existence of positive solutions of semilinear elliptic equations, J. Math. Pures Appl. 61 (1982), 41-63. MR 83h:35039 
5. B. Gidas, W.-M. Ni, and L. Nirenberg, Symmetry and related properties via the maximum principle, Comm. Math. Phys. 68 (1979), 209-243. MR 80h:35043

6. D. Gilbarg and S. N. Trudinger, Elliptic partial differential equations of second order, second edition, Springer-Verlag, Berlin, Heidelberg, New York, and Tokyo, 1983. MR 86c:35035

7. C.-S. Lin, Uniqueness of solutions minimizing the functional $\int_{\Omega}|\nabla u|^{2} /\left(\int_{\Omega} u^{p+1}\right)^{2 / p+1}$ in $R^{2}$, preprint.

8. X. Ren and J. Wei, On a two dimensional elliptic problem with large exponent in nonlinearity, Trans. Amer. Math. Soc. 343 (1994), 749-763. MR 94h:35074

9. Counting peaks of solutions to some quasilinear elliptic equations with large exponents, J. Differential Equations 117 (1995), 28-55.

10. _ Asymptotic behavior of energy solutions to a two dimensional semilinear problem with mixed boundary condition, Nonlinear Anal.: TMA 24 (1995), 587-604. CMP 95:07

School of Mathematics, University of Minnesota, Minneapolis, Minnesota 55455 Current address, X. Ren: Institute for Mathematics \& Applications, University of Minnesota, Minneapolis, Minnesota 55455

E-mail address, X. Ren: ren@ima.umn.edu

Current address, J. Wei: Department of Mathematics, Chinese University of Hong Kong, Shatin, N.T., Hong Kong 